\title{
Symmetry Breaking Soliton, Breather, and Lump Solutions of a Nonlocal Kadomtsev-Petviashvili System
}

\author{
Hong-Yu Wu $\left(\mathbb{D},{ }^{1,2}\right.$ Jin-Xi Fei, ${ }^{1}$ Zheng-Yi Ma $\mathbb{D}^{3},{ }^{3,4}$ Jun-Chao Chen $\mathbb{D}^{3},^{3}$ \\ and Wen-Xiu Ma iD $2,5,6,7,8,9$ \\ ${ }^{1}$ Department of Photoelectric Engineering, Lishui University, Lishui 323000, China \\ ${ }^{2}$ Department of Mathematics and Statistics, University of South Florida, Tampa, FL 33620-5700, USA \\ ${ }^{3}$ Institute of Nonlinear Analysis and Department of Mathematics, Lishui University, Lishui 323000, China \\ ${ }^{4}$ Department of Mathematics, Zhejiang Sci-Tech University, Hangzhou 310018, China \\ ${ }^{5}$ Department of Mathematics, Zhejiang Normal University, Jinhua 321004, China \\ ${ }^{6}$ Department of Mathematics, King Abdulaziz University, Jeddah, Saudi Arabia \\ ${ }^{7}$ School of Mathematics, South China University of Technology, Guangzhou 510640, China \\ ${ }^{8}$ College of Mathematics and Systems Science, Shandong University of Science and Technology, Qingdao 266590, \\ Shandong, China \\ ${ }^{9}$ Department of Mathematical Sciences, North-West University, Mafikeng Campus, Private Bag X2046, \\ Mmabatho 2735, South Africa
}

Correspondence should be addressed to Hong-Yu Wu; why160@126.com and Wen-Xiu Ma; mawx@cas.usf.edu

Received 26 September 2019; Accepted 18 November 2019; Published 10 March 2020

Guest Editor: Sundarapandian Vaidyanathan

Copyright ( 92020 Hong-Yu Wu et al. This is an open access article distributed under the Creative Commons Attribution License, which permits unrestricted use, distribution, and reproduction in any medium, provided the original work is properly cited.

The Kadomtsev-Petviashvili equation is one of the well-studied models of nonlinear waves in dispersive media and in multicomponent plasmas. In this paper, the coupled Alice-Bob system of the Kadomtsev-Petviashvili equation is first constructed via the parity with a shift of the space variable $x$ and time reversal with a delay. By introducing an extended Bäcklund transformation, symmetry breaking soliton, symmetry breaking breather, and symmetry breaking lump solutions for this system are presented through the established Hirota bilinear form. According to the corresponding constants in the involved ansatz function, a few fascinating symmetry breaking structures of the presented explicit solutions are shown.

\section{Introduction}

The localized excitations in nonlinear evolution equations have been studied widely, which were originated from many scientific fields, such as fluid dynamics, plasma physics, superconducting physics, condensed matter physics, and optical problems. Explicitly, the inverse scattering method [1], the Darboux transformation and the Bäcklund transformation $[2,3]$, the Painlevé analysis approach [4-6], the Hirota bilinear method $[7,8]$, and the generalized bilinear method [9] are among important approaches for studying these structures, especially solitary waves and solitons.

Owing to the idea of the parity-time reversal (PT) symmetry, the nonlinear Schrödinger (NLS) equation

$$
\begin{aligned}
i A_{t}+A_{x x} \pm A^{2} B & =0, \\
B=\widehat{f} A & \widehat{P} \widehat{C} A=A^{*}(-x, t),
\end{aligned}
$$

(where the operators $\widehat{P}$ and $\widehat{C}$ are the usual parity and charge conjugation) was introduced and investigated [10]. Based on this, the revolutionary works, which named the Alice-Bob $(\mathrm{AB})$ systems to describe two-place physical problems, were made by Lou recently $[11,12]$. The technical approach originated from the so-called $\widehat{P}-\widehat{T}$ - $\widehat{C}$ principle with $\widehat{P}$ (the parity), $\widehat{T}$ (time reversal), and $\widehat{C}$ (charge conjugation) [11-25]. From this, a general Nth Darboux transformation for the $\mathrm{AB}-\mathrm{mKdV}$ equation was constructed [13]. By using this Darboux transformation, some types of $\widehat{P} \widehat{T}$ symmetry 
breaking solutions including soliton and rogue wave solutions were explicitly obtained. Combined with their Hirota bilinear forms, prohibitions caused by nonlocality for nonlocal Boussinesq-KdV type systems were investigated [14]. The two/four-place nonlocal Kadomtsev-Petviashvili (KP) equation were also explicitly solved for special types of multiple soliton solutions via a $\widehat{P}-\widehat{T}-\widehat{C}$ symmetric-antisymmetric separation approach [15]. From the viewpoint of physical phenomena in climate disasters, a special approximate solution was applied to theoretically capture the salient features of two correlated dipole blocking events in atmospheric dynamical systems and the original two-vortex interaction was given to describe two correlated dipole blocking events with a lifetime through the models established from the nonlinear inviscid dissipative and equivalent barotropic vorticity equation in a $b$-plane [21, 22]. Also, a concrete $\mathrm{AB}-\mathrm{KdV}$ system established from the nonlinear inviscid dissipative and barotropic vorticity equation in a $\beta$-plane channel was applied to the two correlated monople blocking events, which were responsible for the snow disaster in the winter of 2007/2008 that happened in Southern China [18]. Meanwhile, the expression

$$
f=\sum_{\{\nu\}} K_{\{\nu\}} \cosh \left(\frac{1}{2} \sum_{i=1}^{N} v_{i} \xi_{i}\right),
$$

plays a crucial role in constructing analytical group invariant multisoliton solutions of the $\mathrm{AB}$ systems, including the KdV-KP-Toda type, mKdV-sG type, NLS type, and discrete $H_{1}$ type $\mathrm{AB}$ systems $[11-16,18]$.

In this paper, we consider the KP equation (3) as an illustrative example, which is one of the well-studied models of nonlinear waves in dispersive media [26, 27] and in multicomponent plasmas [28]. In the immovable laboratory coordinate frame, it can be presented in the form

$$
\left(u_{t}+c u_{x}+\alpha u u_{x}+\beta u_{x x x}\right)_{x}+\frac{c}{2} u_{y y}=0
$$

where $c$ is the velocity of long linear perturbations and $\alpha$ and $\beta$ are the nonlinear and dispersive coefficients which are determined by specific types of wave and medium properties.

The rest of this paper is organized as follows. In Section 2 , an AB-KP system is constructed based on equation (3) and its Hirota bilinear form is presented through an extended Bäcklund transformation. In Section 3, symmetry breaking soliton, symmetry breaking breather, and symmetry breaking lump solutions are generated through the established Hirota bilinear form, according to the corresponding constants of the involved ansatz function. Some conclusions are given in the final section.

\section{An AB-KP System and Its Bäcklund Transformation and Bilinear Form}

Based on the principle of the $A B$ system in Refs. $[11,12]$, after substituting $u=1 / 2(A+B)$ into equation (3), the AB$\mathrm{KP}$ initial equation is

$$
\begin{aligned}
& A_{x t}+B_{x t}+\frac{1}{2} \alpha\left(A_{x}+B_{x}\right)^{2}+\left[c+\frac{1}{2} \alpha(A+B)\right]\left(A_{x x}+B_{x x}\right) \\
& +\beta\left(A_{x x x x}+B_{x x x x}\right)+\frac{c}{2}\left(A_{y y}+B_{y y}\right)=0,
\end{aligned}
$$

which can be split into the coupled equations

$$
\begin{aligned}
A_{x t} & +\frac{\alpha}{4}\left(A_{x}+B_{x}\right)^{2}+c A_{x x}+\frac{\alpha}{4}(A+B)\left(A_{x x}+B_{x x}\right) \\
& +\beta A_{x x x x}+\frac{c}{2} A_{y y}+G(A, B)=0, \\
B_{x t} & +\frac{\alpha}{4}\left(A_{x}+B_{x}\right)^{2}+c B_{x x}+\frac{\alpha}{4}(A+B)\left(A_{x x}+B_{x x}\right) \\
& +\beta B_{x x x x}+\frac{c}{2} B_{y y}-G(A, B)=0,
\end{aligned}
$$

where $B$ is related to $A$ through $B=\widehat{P}_{s}^{x} \widehat{T}_{d} A=$ $A\left(-x+x_{0}, y,-t+t_{0}\right)\left(\widehat{P}_{s}^{x} \widehat{T}_{d}\right.$ expresses parity with a shift of the space variable $x$ and time reversal with a delay), and $G(A, B)$ is an arbitrary function of $A$ and $B$, but should be $\widehat{P}_{s}^{x} \widehat{T}_{d}$ invariant. That is, $G(A, B)=\widehat{P}_{s}^{x} \widehat{T}_{d} G(A, B)$. Although there are infinitely many functions satisfying this, we take a nontrivial function $G(A, B)$ as

$$
G(A, B)=\frac{\alpha}{2}\left(A_{x}^{2}+A A_{x x}-B_{x}^{2}-B B_{x x}\right)
$$

at present, and equation (5) is reduced to the following ABKP system:

$$
\begin{aligned}
A_{x t} & +\frac{\alpha}{4}\left(A_{x}+B_{x}\right)\left(3 A_{x}-B_{x}\right)+\frac{\alpha}{4}(A-B) B_{x x} \\
+ & {\left[\frac{\alpha}{4}(3 A+B)+c\right] A_{x x}+\beta A_{x x x x}+\frac{c}{2} A_{y y}=0 } \\
B_{x t} & -\frac{\alpha}{4}\left(A_{x}+B_{x}\right)\left(A_{x}-3 B_{x}\right)-\frac{\alpha}{4}(A-B) A_{x x} \\
+ & {\left[\frac{\alpha}{4}(A+3 B)+c\right] B_{x x}+\beta B_{x x x x}+\frac{c}{2} B_{y y}=0 . }
\end{aligned}
$$

In fact, this AB-KP system can also be derived as a special reduction of the coupled $\mathrm{KP}$ system:

$$
\begin{aligned}
& {\left[A_{t}+\left(c_{1} A+c_{2} B\right)_{x}+\left(c_{3} A+c_{4} B\right)_{x x x}+\left(c_{5} A+c_{6} B\right) A_{x}\right.} \\
& \left.\quad+\left(c_{7} A+c_{8} B\right) B_{x}\right]_{x}+c_{9} A_{y y}=0
\end{aligned}
$$

$$
\begin{aligned}
& {\left[B_{t}+\left(c_{1} B+c_{2} A\right)_{x}+\left(c_{3} B+c_{4} A\right)_{x x x}+\left(c_{5} B+c_{6} A\right) B_{x}\right.} \\
& \left.\quad+\left(c_{7} B+c_{8} A\right) A_{x}\right]_{x}+c_{9} B_{y y}=0,
\end{aligned}
$$

by taking the reduction condition $B=\widehat{P}_{s}^{x} \widehat{T}_{d} A=A\left(-x+x_{0}\right.$, $\left.y,-t+t_{0}\right)$ and letting the arbitrary constants $c_{i}(i=1,2, \ldots, 9)$ with 


$$
\begin{aligned}
& c_{1}=c, \\
& c_{2}=c_{4}=0, \\
& c_{3}=\beta, \\
& c_{5}=\frac{3 \alpha}{4}, \\
& c_{6}=c_{7}=\frac{\alpha}{4}, \\
& c_{8}=-\frac{\alpha}{4}, \\
& c_{9}=\frac{c}{2} .
\end{aligned}
$$

Now, we introduce an extended Bäcklund transformation:

$$
\begin{aligned}
& A=\frac{12 \beta}{\alpha}(\ln f)_{x x}+b_{1}(\ln f)_{x x x}+b_{2}(\ln f)_{x x t}, \\
& B=\frac{12 \beta}{\alpha}(\ln f)_{x x}-b_{1}(\ln f)_{x x x}-b_{2}(\ln f)_{x x t},
\end{aligned}
$$

where $b_{1}$ and $b_{2}$ are arbitrary constants and $f \equiv f(x, y, t)$ is a new function of variables $x, y$, and $t$, satisfying the invariant condition

$$
f(x, y, t)=\widehat{P}_{s}^{x} \widehat{T}_{d} f(x, y, t)=f\left(-x+x_{0}, y,-t+t_{0}\right) .
$$

When $b_{1}=0$ and $b_{2}=0$, equation (10) becomes the standard Bäcklund transformation of equation (3). Substituting the transformation equation (10) into equation (7), we obtain a bilinear form of equation (7) as follows:

$$
\left(D_{x} D_{t}+c D_{x}^{2}+\beta D_{x}^{4}+\frac{c}{2} D_{y}^{2}\right)(f \cdot f)=0
$$

where $D_{x}^{4}$ and $D_{y}^{2}$ are the Hirota bilinear derivative operators defined by $[7,8]$

$$
\begin{aligned}
D_{x}^{m} D_{y}^{n} D_{t}^{l}(f \cdot g)= & \left(\frac{\partial}{\partial x}-\frac{\partial}{\partial x^{\prime}}\right)^{m}\left(\frac{\partial}{\partial y}-\frac{\partial}{\partial y^{\prime}}\right)^{n}\left(\frac{\partial}{\partial t}-\frac{\partial}{\partial t^{\prime}}\right)^{l} \\
& \times\left. f(x, y, t) g\left(x^{\prime}, y^{\prime}, t^{\prime}\right)\right|_{x^{\prime}=x, y=y^{\prime}, t^{\prime}=t}
\end{aligned}
$$

According to the properties of the Hirota bilinear operator, equation (12) reads

$$
\begin{aligned}
& 2\left(f f_{x t}-f_{x} f_{t}\right)+2 c\left(f f_{x x}-f_{x}^{2}\right) \\
& \quad+2 \beta\left(f f_{x x x x}-4 f_{x} f_{x x x}+3 f_{x x}^{2}\right)+c\left(f f_{y y}-f_{y}^{2}\right)=0,
\end{aligned}
$$

which is also the Hirota bilinear form of equation (3).

As we know, the Hirota bilinear method is direct and effective for constructing exact solutions, in which a given nonlinear equation is converted to a bilinear form through an appropriate transformation. With different types of ansatz for the auxiliary function, a variety of soliton, rational, and periodic solutions of the nonlinear equation can be derived.

\section{Symmetry Breaking Soliton, Breather, and Lump Solutions}

In this section, we turn our attention to the Hirota bilinear form (12) of the AB-KP systems (7a) and (7b) to derive symmetry breaking soliton, symmetry breaking breather, and symmetry breaking lump solutions.

3.1. Symmetry Breaking Soliton Solutions. Based on the bilinear form (12), we can first determine symmetry breaking soliton solutions through the Bäcklund transformation (10) of the AB-KP systems ( $7 \mathrm{a})$ and (7b) with the function $f$ being written as a summation of some special functions $[11-16,18]$ :

$$
\begin{aligned}
& f=f_{N}=\sum_{\{v\}} K_{\{v\}} \cosh \left(\frac{1}{2} \sum_{i=1}^{N} v_{i} \xi_{i}\right), \\
& \xi_{i}=k_{i}\left[\left(x-\frac{x_{0}}{2}\right)+p_{i} y-\left(c+k_{i}^{2} \beta+\frac{c p_{i}^{2}}{2}\right)\left(t-\frac{t_{0}}{2}\right)\right]+\eta_{i 0},
\end{aligned}
$$

where $\quad\{\nu\}=\left\{v_{1}, v_{2}, \ldots, v_{N}\right\}$, with $\quad v_{i}= \pm 1$, and $k_{i}, p_{i}, \eta_{i 0}(i=1,2, \ldots, N), c, x_{0}$, and $t_{0}$ are undetermined constants, while

$$
K_{\{\nu\}}=\prod_{i<j}^{N} \sqrt{c\left(p_{i}-p_{j}\right)^{2}-6 \beta\left(k_{i}-v_{i} \nu_{j} k_{j}\right)^{2}} .
$$

For $N=1$, equation (15) takes the form

$$
\begin{aligned}
& f=f_{1}=\cosh \left(\frac{\xi_{1}}{2}\right), \\
& \xi_{1}=k_{1}\left[\left(x-\frac{x_{0}}{2}\right)+p_{1} y-\left(c+k_{1}^{2} \beta+\frac{c p_{1}^{2}}{2}\right)\left(t-\frac{t_{0}}{2}\right)\right]+\eta_{10} .
\end{aligned}
$$

However, the invariant condition (11) of this function $f$ (17) is not satisfied for the constants $k_{1}, p_{1}, c, x_{0}, t_{0}$, and $\eta_{10}$ being not all zero. It means that it is impossible to get any nontrivial symmetry breaking single soliton solution of equation (12) through (10).

The same circumstance happens when $N=3$, in which the function $f$ of equation (15) is

$$
\begin{aligned}
f= & f_{3}=K_{\{\}} \cosh \left\{\frac{1}{2}\left(\xi_{1}+\xi_{2}+\xi_{3}\right)\right\} \\
& +K_{\{1\}} \cosh \left\{\frac{1}{2}\left(\xi_{1}-\xi_{2}-\xi_{3}\right)\right\} \\
& +K_{\{2\}} \cosh \left\{\frac{1}{2}\left(\xi_{1}-\xi_{2}+\xi_{3}\right)\right\} \\
& +K_{\{3\}} \cosh \left\{\frac{1}{2}\left(\xi_{1}+\xi_{2}-\xi_{3}\right)\right\},
\end{aligned}
$$


where

$$
\begin{gathered}
K_{\{\}}=a_{12}^{-} a_{13}^{-} a_{23}^{-}, \\
K_{\{1\}}=a_{12}^{+} a_{13}^{+} a_{23}^{-}, \\
K_{\{2\}}=a_{12}^{+} a_{13}^{-} a_{23}^{+}, \\
K_{\{3\}}=a_{12}^{-} a_{13}^{+} a_{23}^{+}, \\
\xi_{i}=k_{i}\left[\left(x-\frac{x_{0}}{2}\right)+p_{i} y-\left(c+k_{i}^{2} \beta+\frac{c p_{i}^{2}}{2}\right)\left(t-\frac{t_{0}}{2}\right)\right]+\eta_{i 0} \\
a_{i j}^{ \pm}=\sqrt{c\left(p_{i}-p_{j}\right)^{2}-6 \beta\left(k_{i} \pm k_{j}\right)^{2}}, \quad i, j=1,2,3 .
\end{gathered}
$$

Furthermore, one can verify that, for any odd $N=2 n-1$ ( $n$ is a positive integer), the function $f(15)$ does not satisfy the invariant condition in equation (11). In other words, symmetry breaking soliton solutions of odd orders for the AB-KP systems (7a) and (7b) are prohibited.

For $N=2$, equation (15) becomes

$$
f=f_{2}=K_{\{\}} \cosh \left(\frac{\left(\xi_{1}+\xi_{2}\right)}{2}\right)+K_{\{1\}} \cosh \left(\frac{\left(\xi_{1}-\xi_{2}\right)}{2}\right) \text {, }
$$

where

$$
\begin{aligned}
K_{\{\}} & =\sqrt{c\left(p_{1}-p_{2}\right)^{2}-6 \beta\left(k_{1}-k_{2}\right)^{2}}, \\
K_{\{1\}} & =\sqrt{c\left(p_{1}-p_{2}\right)^{2}-6 \beta\left(k_{1}+k_{2}\right)^{2}} .
\end{aligned}
$$

By fixing the real parameters,

$$
\begin{gathered}
k_{2}= \pm k_{1}, \\
p_{2}=-p_{1}, \\
\eta_{20}=\mp \eta_{10},
\end{gathered}
$$

the invariant condition in equation (11) is satisfied. Correspondingly, by writing

$$
\begin{aligned}
f= & f_{2}=\sqrt{c p_{1}^{2}} \cosh \left\{k_{1}\left[\left(x-\frac{x_{0}}{2}\right)-\left(c+k_{1}^{2} \beta+\frac{c p_{1}^{2}}{2}\right)\left(t-\frac{t_{0}}{2}\right)\right]\right\} \\
& +\sqrt{c p_{1}^{2}-6 \beta k_{1}^{2}} \cosh \left(k_{1} p_{1} y+\eta_{10}\right),
\end{aligned}
$$

a symmetry breaking two-soliton solution of equations (7a) and (7b) are expressed as

$$
\begin{aligned}
& A=\frac{12 \beta}{\alpha}\left(\ln f_{2}\right)_{x x}+b_{1}\left(\ln f_{2}\right)_{x x x}+b_{2}\left(\ln f_{2}\right)_{x x t}, \\
& B=\frac{12 \beta}{\alpha}\left(\ln f_{2}\right)_{x x}-b_{1}\left(\ln f_{2}\right)_{x x x}-b_{2}\left(\ln f_{2}\right)_{x x t} .
\end{aligned}
$$

Figure 1 shows the symmetry breaking two-soliton structure of solution (24) with the parameters being taken as

$$
\begin{aligned}
& \alpha=k_{1}=p_{1}=1, \\
& \beta=\frac{1}{6}, \\
& c=4, \\
& x_{0}=t_{0}=\eta_{10}=0 .
\end{aligned}
$$

Meanwhile, Figure 1(a) describes a standard two-soliton structure $\left(b_{1}=b_{2}=0\right)$ for solution (24) at time $t=0$. Under this special condition, the solution $A$ coincides with the solution $B$ exactly. Figures 1(b) and 1(c) are two symmetry breaking two-soliton structures for solution (24) with the selected parameters $b_{1}=b_{2}=10$ at time $t=0$. Obviously, Figure 1(c) depicts a reversal structure of Figure 1(b) by the solution $B$ which is $\widehat{P}_{s}^{x} \widehat{T}_{d}$ symmetry of the solution $A$ for the $\mathrm{AB}-\mathrm{KP}$ systems (7a) and (7b). This corresponds to the phenomenon that the shifted parity and delayed time reversal are applied to describe two-place events $[11,12]$. These structures are realized via the symbolic computation software Maple efficiently.

For $N=4$, the function $f$ of equation (15) can be rewritten regularly as

$$
\begin{aligned}
f= & f_{4}=K_{\{\}} \cosh \left\{\frac{1}{2}\left(\xi_{1}+\xi_{2}+\xi_{3}+\xi_{4}\right)\right\}+K_{\{1\}} \cosh \left\{\frac{1}{2}\left(-\xi_{1}+\xi_{2}+\xi_{3}+\xi_{4}\right)\right\} \\
& +K_{\{2\}} \cosh \left\{\frac{1}{2}\left(\xi_{1}-\xi_{2}+\xi_{3}+\xi_{4}\right)\right\}+K_{\{3\}} \cosh \left\{\frac{1}{2}\left(\xi_{1}+\xi_{2}-\xi_{3}+\xi_{4}\right)\right\} \\
& +K_{\{4\}} \cosh \left\{\frac{1}{2}\left(\xi_{1}+\xi_{2}+\xi_{3}-\xi_{4}\right)\right\}+K_{\{23\}} \cosh \left\{\frac{1}{2}\left(\xi_{1}-\xi_{2}-\xi_{3}+\xi_{4}\right)\right\} \\
& +K_{\{24\}} \cosh \left\{\frac{1}{2}\left(\xi_{1}-\xi_{2}+\xi_{3}-\xi_{4}\right)\right\}+K_{\{34\}} \cosh \left\{\frac{1}{2}\left(\xi_{1}+\xi_{2}-\xi_{3}-\xi_{4}\right)\right\}
\end{aligned}
$$




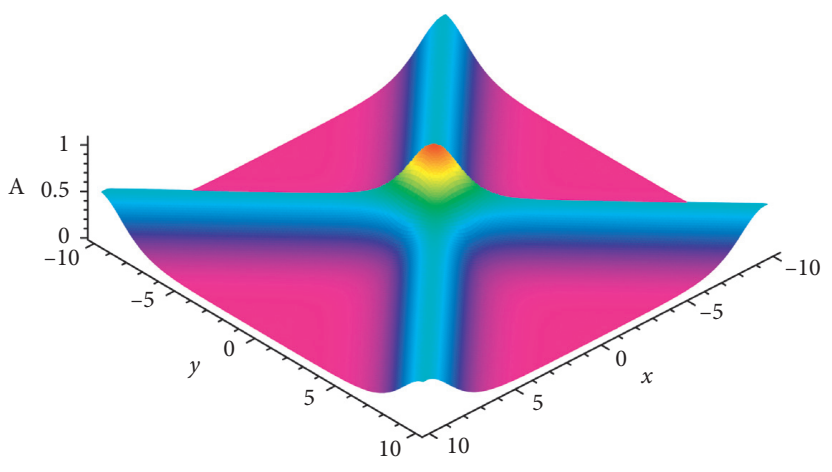

(a)

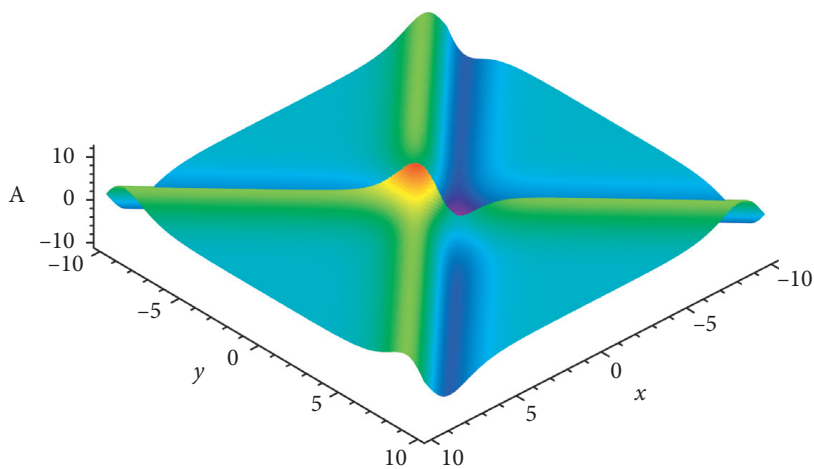

(b)

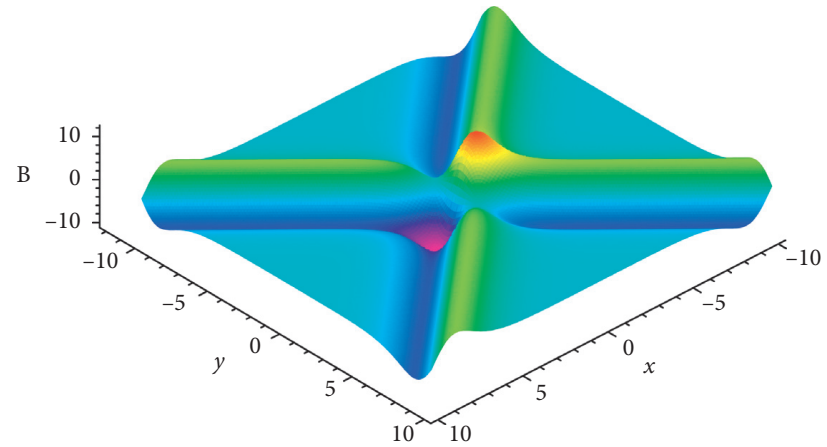

(c)

FIgURE 1: The symmetry breaking two-soliton solution (24) of the AB-KP systems (7a) and (7b), with the selecting parameters $b_{1}$ and $b_{2}$ are (a) $b_{1}=b_{2}=0$ and (b) and (c) $b_{1}=b_{2}=10$, at time $t=0$.

where

$$
\begin{aligned}
K_{\{\}} & =a_{12}^{-} a_{13}^{-} a_{14}^{-} a_{23}^{-} a_{24}^{-} a_{34}^{-}, \\
K_{\{1\}} & =a_{12}^{+} a_{13}^{+} a_{14}^{+} a_{23}^{-} a_{24}^{-} a_{34}^{-}, \\
K_{\{2\}} & =a_{12}^{+} a_{13}^{-} a_{14}^{-} a_{23}^{+} a_{24}^{+} a_{34}^{-}, \\
K_{\{3\}} & =a_{12}^{-} a_{13}^{+} a_{14}^{-} a_{23}^{+} a_{24}^{-} a_{34}^{+}, \\
K_{\{4\}} & =a_{12}^{-} a_{13}^{-} a_{14}^{+} a_{23}^{-} a_{24}^{+} a_{34}^{+}, \\
K_{\{23\}} & =a_{12}^{+} a_{13}^{+} a_{14}^{-} a_{23}^{-} a_{24}^{+} a_{34}^{+}, \\
K_{\{24\}} & =a_{12}^{+} a_{13}^{-} a_{14}^{+} a_{23}^{+} a_{24}^{-} a_{34}^{+}, \\
K_{\{34\}} & =a_{12}^{-} a_{13}^{+} a_{14}^{+} a_{23}^{+} a_{24}^{+} a_{34}^{-}, \\
\xi_{i} & =k_{i}\left[\left(x-\frac{x_{0}}{2}\right)^{+} p_{i} y-\left(c+k_{i}^{2} \beta+\frac{c p_{i}^{2}}{2}\right)\left(t-\frac{t_{0}}{2}\right)\right]+\eta_{i 0}, \\
a_{i j}^{ \pm} & =\sqrt{c\left(p_{i}-p_{j}\right)^{2}-6 \beta\left(k_{i} \pm k_{j}\right)^{2},} \quad i, j=1,2,3,4 .
\end{aligned}
$$

After finishing some detailed analysis, there are two independent real parameter selections of the symmetry breaking four-soliton solution for (7a) and (7b), which are

$$
\begin{aligned}
& k_{3}= \pm k_{1}, \\
& k_{4}= \pm k_{2}, \\
& p_{3}=-p_{1}, \\
& p_{4}=-p_{2}, \\
& \eta_{30}=\mp \eta_{10}, \\
& \eta_{40}=\mp \eta_{20},
\end{aligned}
$$

with $B=\widehat{P}_{s} \widehat{T}_{d} A=A\left(-x+x_{0}, y=y,-t+t_{0}\right)$. Based on these restrictions in (28), we have

$$
\begin{gathered}
a_{23}^{ \pm}=a_{14}^{ \pm}, \\
a_{34}^{ \pm}=a_{12}^{ \pm}, \\
K_{\{3\}}=K_{\{1\}}, \\
K_{\{4\}}=K_{\{2\}} .
\end{gathered}
$$

At this time, the symmetry breaking four-soliton solution of the AB-KP systems (7a) and (7b) is

$$
\begin{aligned}
& A=\frac{12 \beta}{\alpha}\left(\ln f_{4}\right)_{x x}+b_{1}\left(\ln f_{4}\right)_{x x x}+b_{2}\left(\ln f_{4}\right)_{x x t}, \\
& B=\frac{12 \beta}{\alpha}\left(\ln f_{4}\right)_{x x}-b_{1}\left(\ln f_{4}\right)_{x x x}-b_{2}\left(\ln f_{4}\right)_{x x t},
\end{aligned}
$$


where

$$
\begin{aligned}
f_{4}= & K_{\{\}} \cosh \left(\zeta_{1}^{+}+\zeta_{2}^{+}\right)+K_{\{1\}}\left[\cosh \left(\zeta_{1}^{-}+\zeta_{2}^{+}\right)+\cosh \left(\zeta_{1}^{-}-\zeta_{2}^{+}\right)\right] \\
& +K_{\{2\}}\left[\cosh \left(\zeta_{1}^{+}+\zeta_{2}^{-}\right)+\cosh \left(\zeta_{1}^{+}-\zeta_{2}^{-}\right)\right] \\
& +K_{\{23\}} \cosh \left(\zeta_{1}^{-}-\zeta_{2}^{-}\right)+K_{\{24\}} \cosh \left(\zeta_{1}^{+}-\zeta_{2}^{+}\right) \\
& +K_{\{34\}} \cosh \left(\zeta_{1}^{-}+\zeta_{2}^{-}\right)
\end{aligned}
$$

with

$$
\begin{aligned}
& \zeta_{1}^{ \pm}=\frac{1}{2}\left(\xi_{1} \pm \xi_{3}\right), \\
& \zeta_{2}^{ \pm}=\frac{1}{2}\left(\xi_{2} \pm \xi_{4}\right) .
\end{aligned}
$$

If setting $\alpha=k_{1}=p_{1}=1, \beta=1 / 6, c=-5, k_{2}=-p_{2}=6 / 5$, and $x_{0}=t_{0}=\eta_{10}=\eta_{20}=0$, we can depict the abovementioned symmetry breaking four-soliton structure in (Figure 2 ). The similar situation is as follows: Figure $2(a)$ is the standard foursoliton structure $\left(b_{1}=b_{2}=0\right)$ for the solution $A=B$ at time $t=0$. Figures 2(b) and 2(c) are two symmetry breaking foursoliton structures for the solution $A$ and $B$, respectively, with the selected parameters $b_{1}=b_{2}=10$ at time $t=0$.

3.2. Symmetry Breaking Breather Solutions. When taking $p_{1}=p_{0} I$ ( $p_{0}$ is a real constant and $I$ is the imaginary unit, $\left.I^{2}=-1\right)$, a symmetry breaking breather solution of the AB$\mathrm{KP}$ systems (7a) and (7b) can read

$$
\begin{aligned}
& A=\frac{12 \beta}{\alpha}\left(\ln f_{b}\right)_{x x}+b_{1}\left(\ln f_{b}\right)_{x x x}+b_{2}\left(\ln f_{b}\right)_{x x t}, \\
& B=\widehat{P}_{s}^{x} \widehat{T}_{d} A=A\left(-x+x_{0}, y,-t+t_{0}\right),
\end{aligned}
$$

with the ansatz function

$$
\begin{aligned}
f= & f_{b}=\sqrt{-c p_{0}^{2}} \cosh \left\{k_{1}\left[\left(x-\frac{x_{0}}{2}\right)-\left(c+k_{1}^{2} \beta-\frac{c p_{0}^{2}}{2}\right)\left(t-\frac{t_{0}}{2}\right)\right]\right\} \\
& +\sqrt{-c p_{0}^{2}-6 \beta k_{1}^{2}} \cosh \left(k_{1} p_{0} I y+\eta_{10}\right),
\end{aligned}
$$

from equation (23).

By some constraints to the parameters in this solution, a family of analytical breather solutions can be generated. For example, when taking the real constants

$$
\begin{aligned}
c & =-4, \\
\alpha & =k_{1}=1, \\
\beta & =\frac{1}{6}, \\
p_{0} & =2, \\
x_{0} & =t_{0}=\eta_{10}=0,
\end{aligned}
$$

equation (34) becomes

$$
f=f_{b}=4 \cosh \left(x-\frac{25}{6} t\right)+\sqrt{15} \cos (2 y)
$$

according to Euler's formula. This function indicates that the solution has two wave components, that is, a regular solitary wave with the propagating speed $-25 / 6$ and a periodic wave with period $\pi$. Figure 3 is a density plot of the breathers defined by equation (36) with the parameters in (35). Figure 3(a) is the standard first-order breather structure $\left(b_{1}=\right.$ $\left.b_{2}=0\right)$ for the solution $A=B=8(\sqrt{15} \cos (2 y) \cosh x+4) /$ $(4 \cosh x+\sqrt{15} \cos (2 y))^{2}$ at time $t=0$. Figures 3(b) and 3(c) are two symmetry breaking breather structures for the solution $A, B=8(\sqrt{15} \cos (2 y) \cosh x+4) /(4 \cosh x+\sqrt{15} \cos$ $(2 y))^{2} \pm 95 \sinh x\left(32+4 \sqrt{15} \cos (2 y) \cosh x-15 \cos ^{2}(2 y)\right) /$ $(4 \cosh x+\sqrt{15} \cos (2 y))^{3}$, with the selected parameters $b_{1}=$ $b_{2}=10$ at time $t=0$. As these solutions combine the trigonometric cosine function with hyperbolic sine/cosine functions, the property of these functions describes the symmetry breaking breather structures [29, 30].

In the abovementioned situation, when taking the constants

$$
\begin{aligned}
c & =-4, \\
\alpha & =1, \\
\beta & =\frac{1}{6}, \\
k_{1} & =I, \\
p_{0} & =2, \\
x_{0} & =t_{0}=\eta_{10}=0,
\end{aligned}
$$

equation (34) has the expression

$$
f=f_{b}=\sqrt{16} \cos \left(x-\frac{23}{6} t\right)+\sqrt{17} \cosh (2 y) .
$$

Figure 4 is a density plot of the breathers described according to equation (38) under the parameter selection (37). That is, when the parameter $k_{1}$ also takes the imaginary unit $I$, the $x$-periodic symmetry breaking breathers of the AB-KP systems (7a) and (7b) are formed.

The abovementioned idea can be extended to solution (30). After setting the parameters

$$
\begin{aligned}
c & =-5, \\
\alpha & =k_{1}=1, \\
\beta & =\frac{1}{6}, \\
k_{2} & =\frac{1}{2}, \\
p_{1} & =I, \\
p_{2} & =-2 I, \\
x_{0} & =t_{0}=\eta_{10}=\eta_{20}=0, \\
c & =-4, \\
\alpha & =1, \\
\beta & =\frac{1}{6}, \\
k_{1} & =p_{1}=-p_{2}=I, \\
k_{2} & =-2 I, \\
x_{0} & =t_{0}=\eta_{10}=\eta_{20}=0,
\end{aligned}
$$




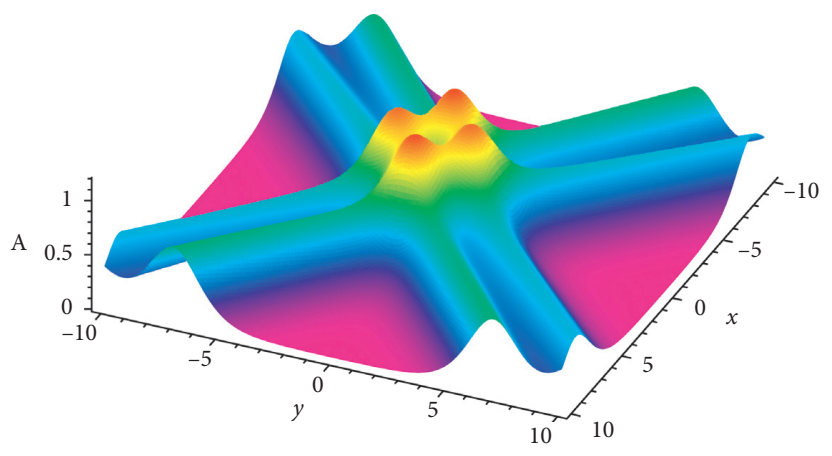

(a)

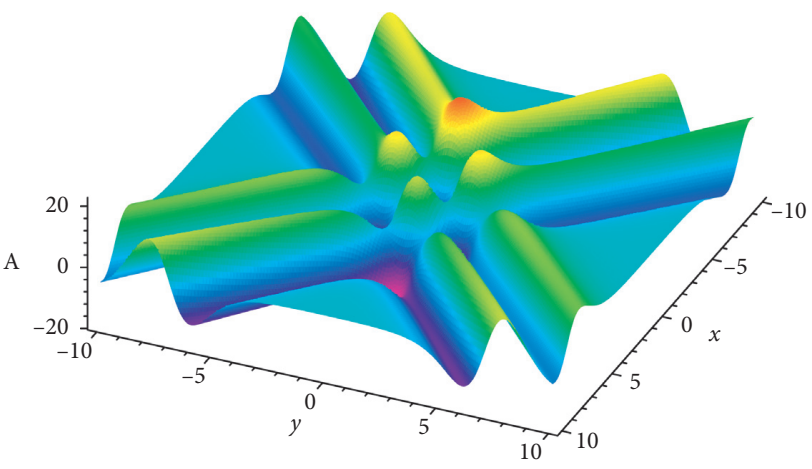

(b)

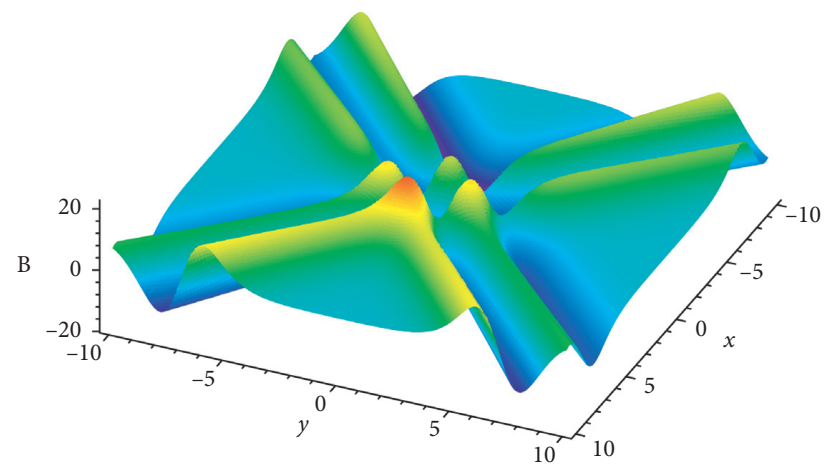

(c)

Figure 2: The symmetry breaking four-soliton solution (30) of the AB-KP systems (7a) and (7b), with the selecting parameters $b_{1}$ and $b_{2}$ are (a) $b_{1}=b_{2}=0$ and (b) and (c) $b_{1}=b_{2}=10$, at time $t=0$.

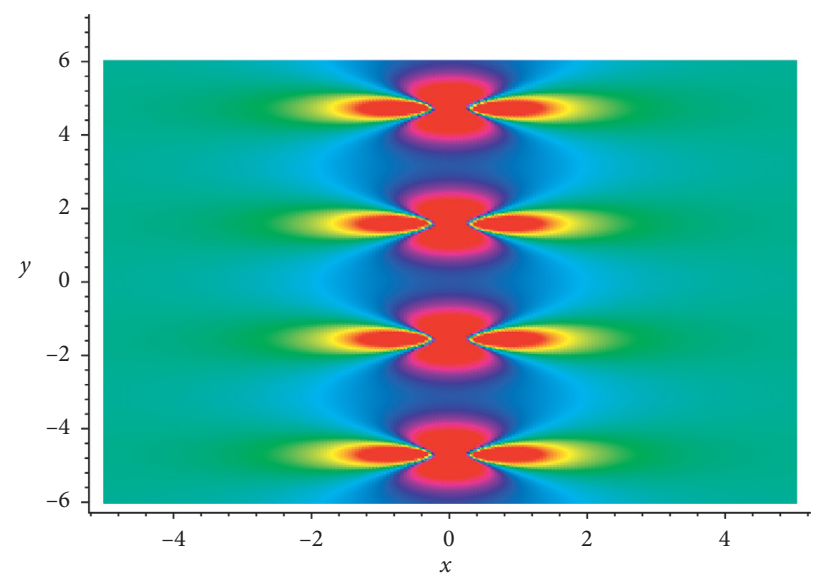

(a)

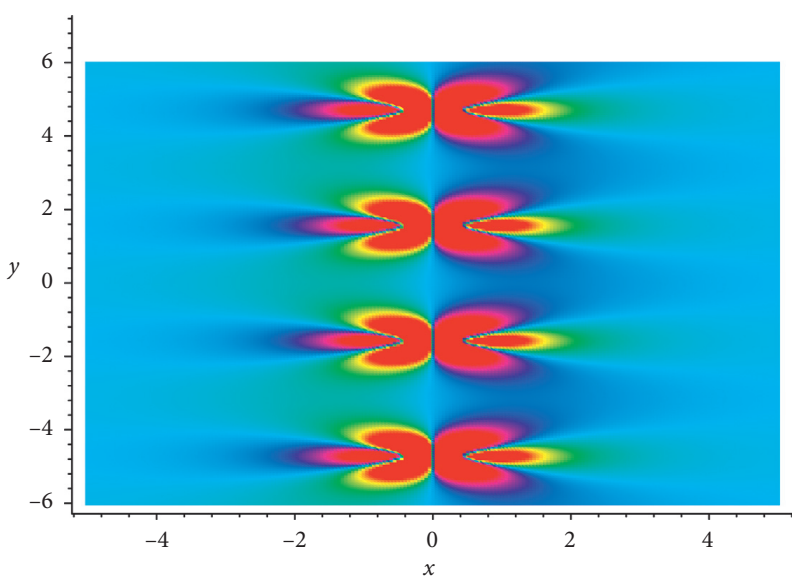

(b)

FIgUre 3: Continued. 


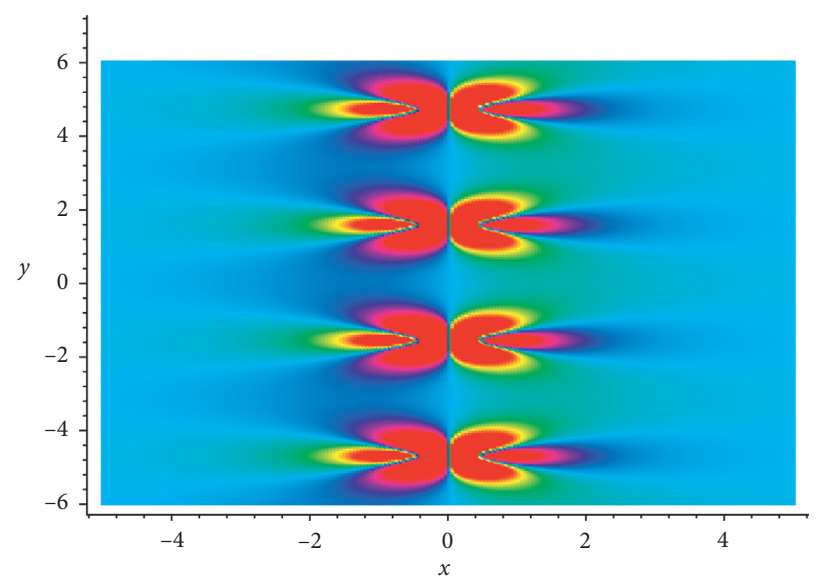

(c)

FIgURE 3: The density plot of the $y$-periodic symmetry breaking breathers of the AB-KP systems (7a) and (7b), with the selected parameters $b_{1}$ and $b_{2}$ are (a) $b_{1}=b_{2}=0$ and (b) and (c) $b_{1}=b_{2}=10$, at time $t=0$.

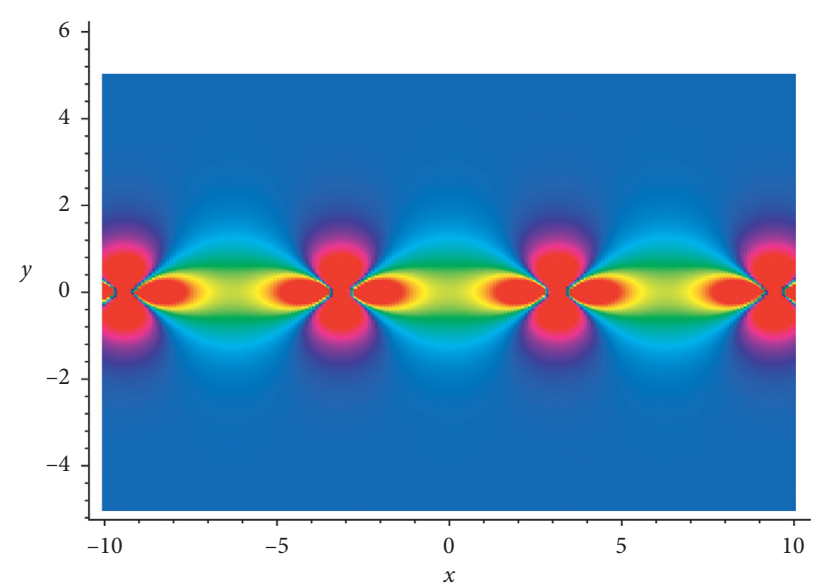

(a)

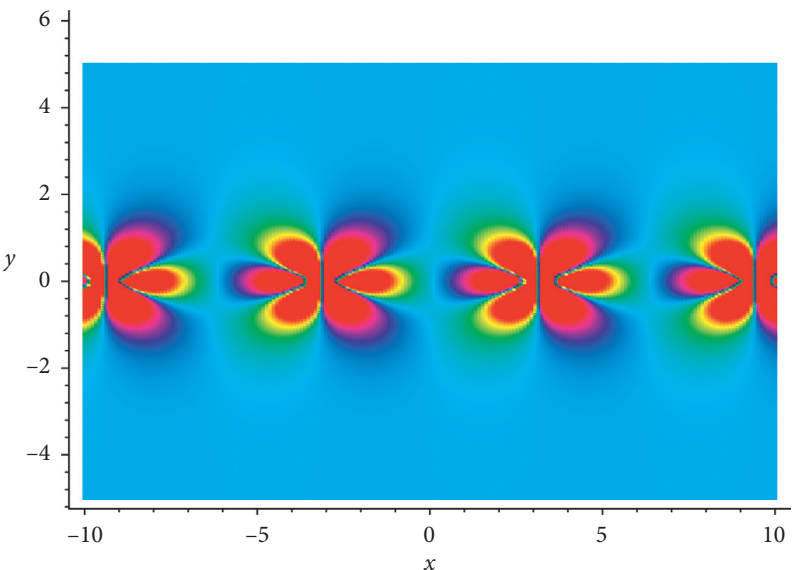

(b)

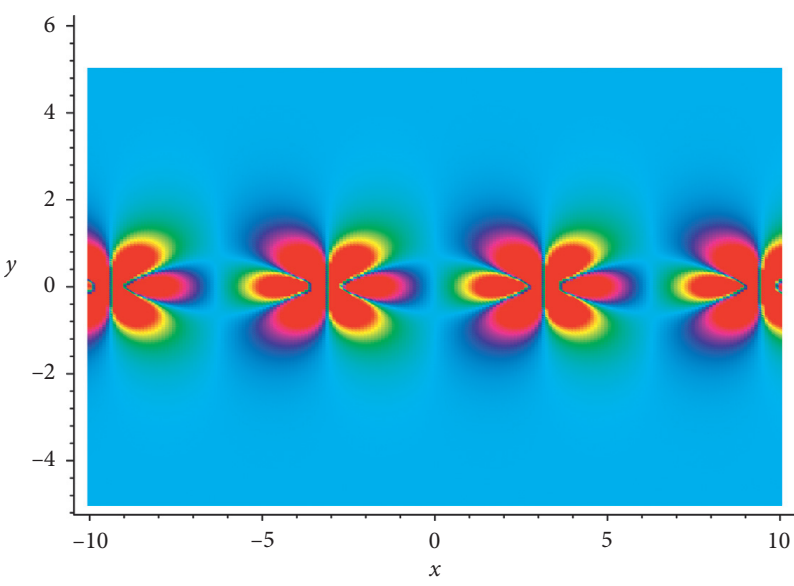

(c)

Figure 4: The density plot of the $x$-periodic symmetry breaking breather of the AB-KP systems (7a) and (7b), with the selected parameters $b_{1}$ and $b_{2}$ are (a) $b_{1}=b_{2}=0$ and (b) and (c) $b_{1}=b_{2}=10$, at time $t=0$.

the $y$-periodic and $x$-periodic second-order breather solutions can be derived, which are symmetry breaking (Figures 5 and 6, respectively).
3.3. Symmetry Breaking Lump Solutions. As we know, the lump solution is expressed by the rational function which is localized in all directions in the space. Based on the long-wave 


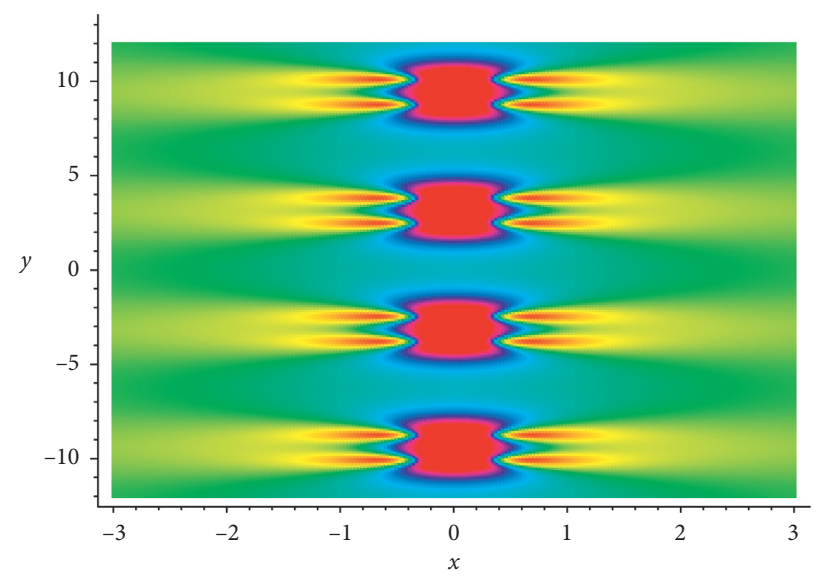

(a)

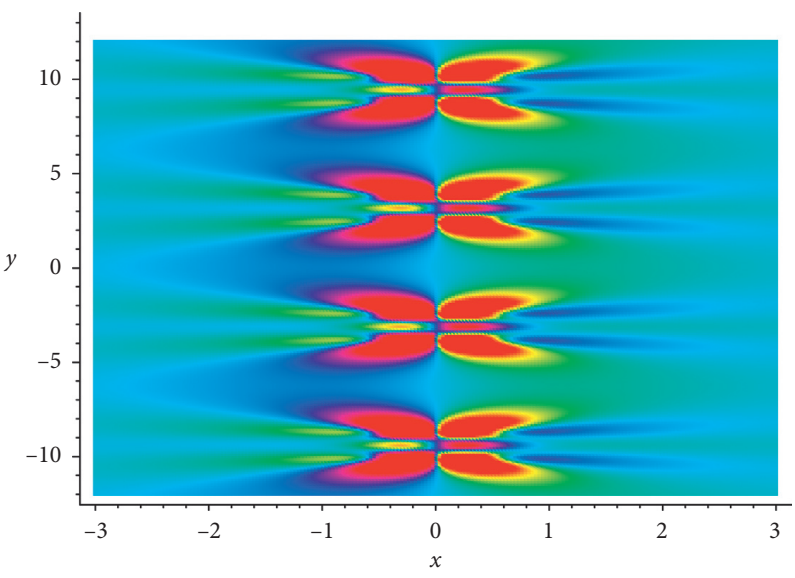

(b)

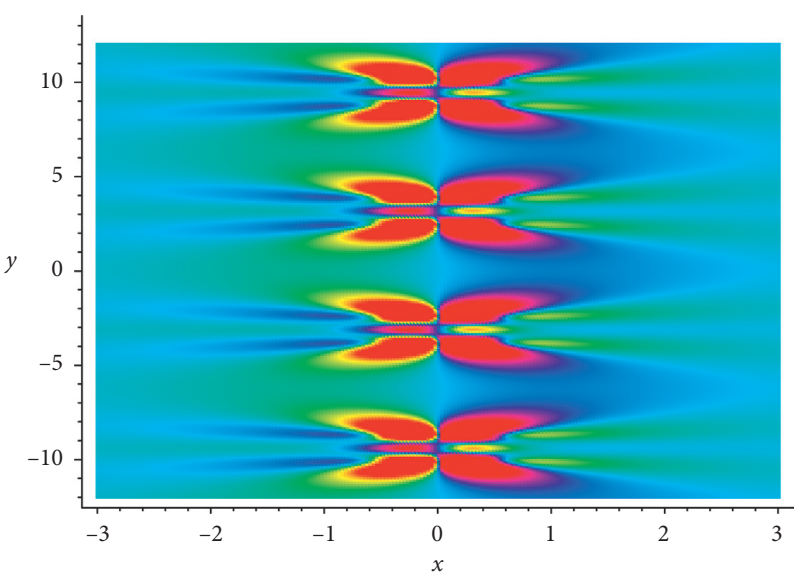

(c)

FIGURE 5: The $y$-periodic symmetry breaking density plot of the second-order breathers of the AB-KP systems (7a) and (7b), with the selected parameters $b_{1}$ and $b_{2}$ are (a) $b_{1}=b_{2}=0$ and (b) and (c) $b_{1}=b_{2}=10$, at time $t=0$.

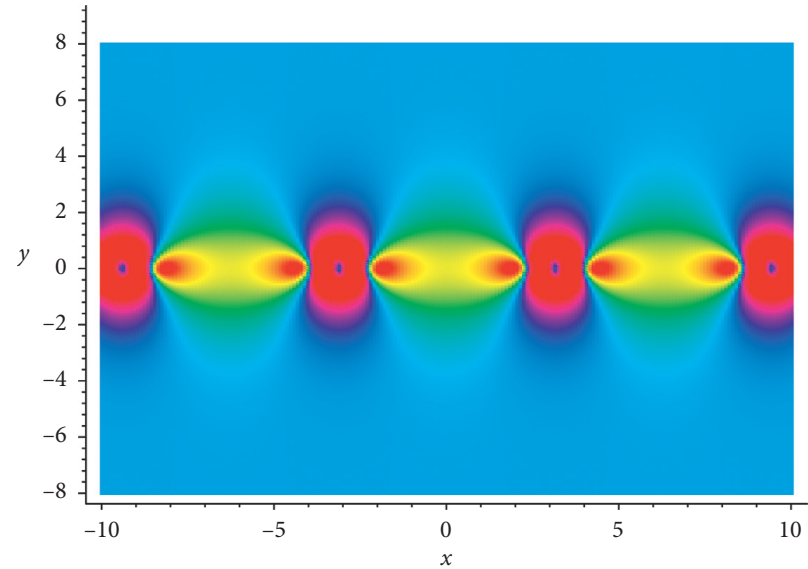

(a)

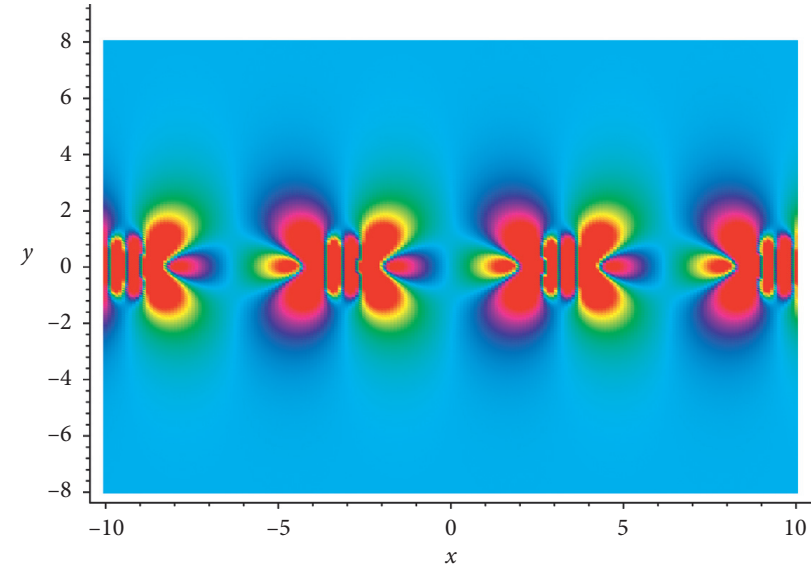

(b)

Figure 6: Continued. 


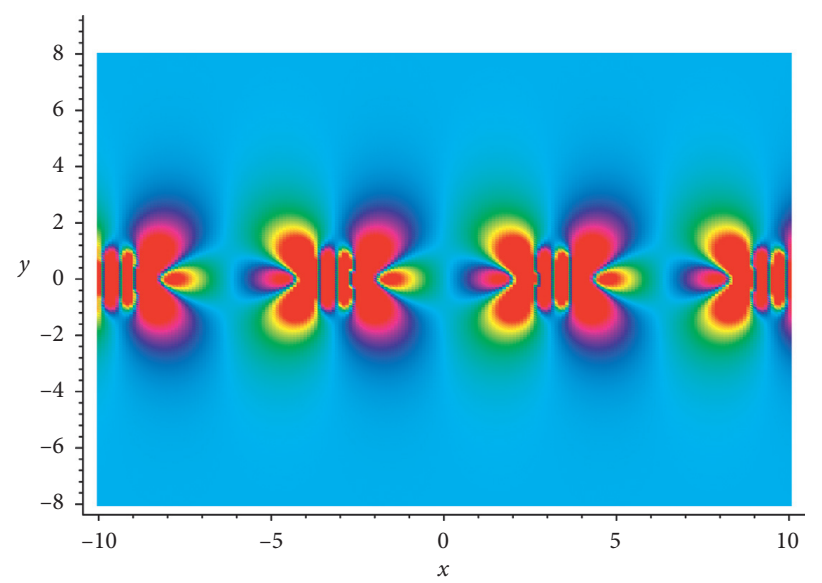

(c)

Figure 6: The $x$-periodic symmetry breaking density plot of the second-order breathers of the AB-KP systems (7a) and (7b), with the selected parameters $b_{1}$ and $b_{2}$ are (a) $b_{1}=b_{2}=0$ and (b) and (c) $b_{1}=b_{2}=10$, at time $t=0$.

limit idea of generating lump solutions to nonlinear equations, we derive this kind of solutions of the AB-KP systems (7a) and (7b) by taking a long-wave limit. After putting

$$
\begin{aligned}
k_{1} & =\delta_{1} \epsilon_{1}, \\
k_{2} & =\delta_{2} \epsilon_{2}, \\
\eta_{10} & =\eta_{20}=I \pi,
\end{aligned}
$$

into the bilinear function (20) and setting the limit $\epsilon_{1} \longrightarrow 0, \epsilon_{2} \longrightarrow 0$, the function $f$ can be obtained:

$$
\begin{aligned}
f= & f_{l}=\delta_{1} \delta_{2}\left[x-\frac{x_{0}}{2}+p_{1} y-c\left(1+\frac{p_{1}^{2}}{2}\right)\left(t-\frac{t_{0}}{2}\right)\right] \\
& \cdot\left[x-\frac{x_{0}}{2}+p_{2} y-c\left(1+\frac{p_{2}^{2}}{2}\right)\left(t-\frac{t_{0}}{2}\right)\right]+\frac{24 \delta_{1} \delta_{2} \beta}{c\left(p_{1}-p_{2}\right)^{2}} .
\end{aligned}
$$

When letting

$$
\begin{aligned}
& p_{1}=\frac{a_{2}+a_{5} I}{\delta_{1}}, \\
& p_{2}=\frac{a_{2}-a_{5} I}{\delta_{2}}, \\
& \delta_{1}=a_{1}+a_{4} I, \\
& \delta_{2}=a_{1}-a_{4} I,
\end{aligned}
$$

equation (41) can be arranged in

$$
\begin{aligned}
& f=f_{l}=g^{2}+h^{2}+a_{7}, \\
& g=a_{1}\left(x-\frac{x_{0}}{2}\right)+a_{2} y+a_{3}\left(t-\frac{t_{0}}{2}\right), \\
& h=a_{4}\left(x-\frac{x_{0}}{2}\right)+a_{5} y+a_{6}\left(t-\frac{t_{0}}{2}\right),
\end{aligned}
$$

where $a_{i}(1 \leq i \leq 7)$ are all real parameters to be determined. Note that the function $f$ in equation (43) is positive if the parameter $a_{7}>0$. Combining equation (43) with equations (11) and (12), the constraining relations of these parameters are

$$
\begin{aligned}
& a_{3}=\frac{a_{1} c\left(a_{2}^{2}-2 a_{4}^{2}\right)}{2 a_{4}^{2}}, \\
& a_{5}=-\frac{a_{1} a_{2}}{a_{4}}, \\
& a_{6}=\frac{c\left(a_{2}^{2}-2 a_{4}^{2}\right)}{2 a_{4}}, \\
& a_{7}=-\frac{6 a_{4}^{2} \beta\left(a_{1}^{2}+a_{4}^{2}\right)}{a_{2}^{2} c},
\end{aligned}
$$

where all the denominators are nonzero.

This time, solution (10) becomes

$$
\begin{aligned}
A= & \frac{12 \beta}{\alpha}\left[\ln \left(g^{2}+h^{2}-\frac{6 a_{4}^{2} \beta\left(a_{1}^{2}+a_{4}^{2}\right)}{a_{2}^{2} c}\right)\right]_{x x} \\
& +b_{1}\left[\ln \left(g^{2}+h^{2}-\frac{6 a_{4}^{2} \beta\left(a_{1}^{2}+a_{4}^{2}\right)}{a_{2}^{2} c}\right)\right]_{x x x} \\
& +b_{2}\left[\ln \left(g^{2}+h^{2}-\frac{6 a_{4}^{2} \beta\left(a_{1}^{2}+a_{4}^{2}\right)}{a_{2}^{2} c}\right)\right]_{x x t}, \\
B= & \frac{12 \beta}{\alpha}\left[\ln \left(g^{2}+h^{2}-\frac{6 a_{4}^{2} \beta\left(a_{1}^{2}+a_{4}^{2}\right)}{a_{2}^{2} c}\right)\right]_{x x} \\
& -b_{1}\left[\ln \left(g^{2}+h^{2}-\frac{6 a_{4}^{2} \beta\left(a_{1}^{2}+a_{4}^{2}\right)}{a_{2}^{2} c}\right)\right]_{x x x} \\
& -b_{2}\left[\ln \left(g^{2}+h^{2}-\frac{6 a_{4}^{2} \beta\left(a_{1}^{2}+a_{4}^{2}\right)}{a_{2}^{2} c}\right)\right]_{x x t},
\end{aligned}
$$




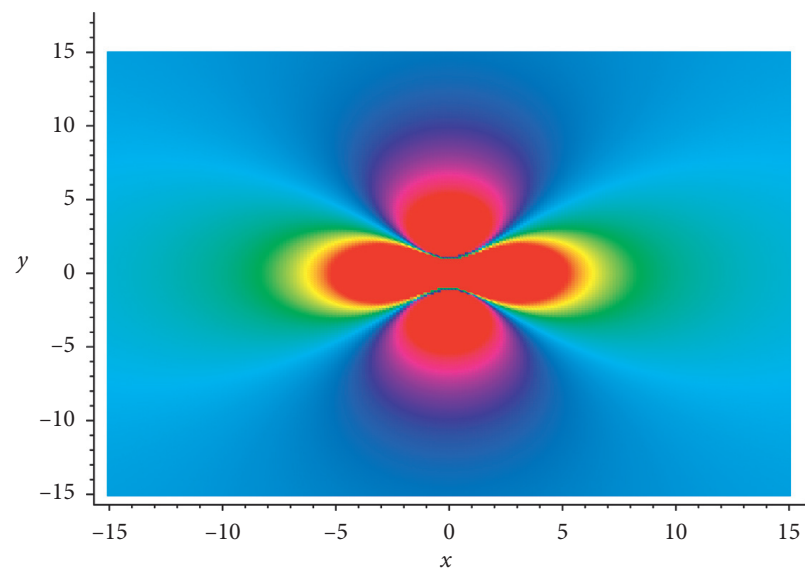

(a)

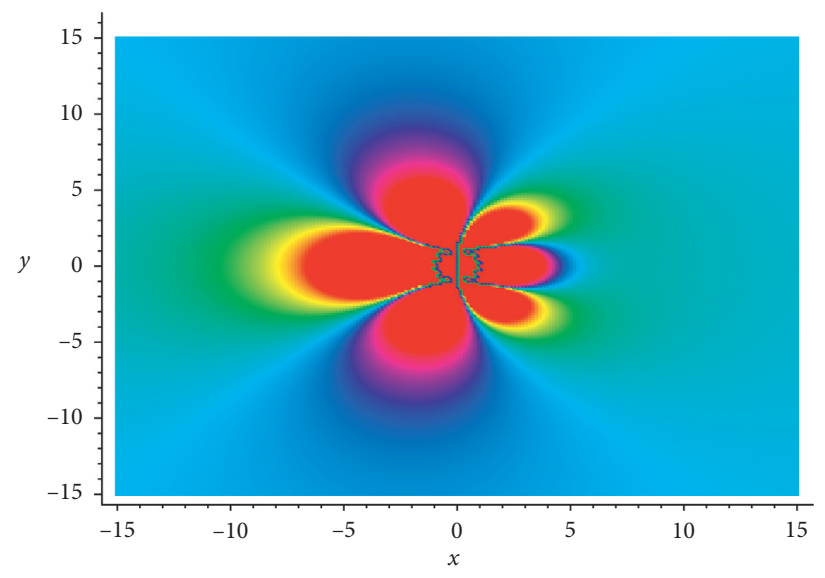

(b)

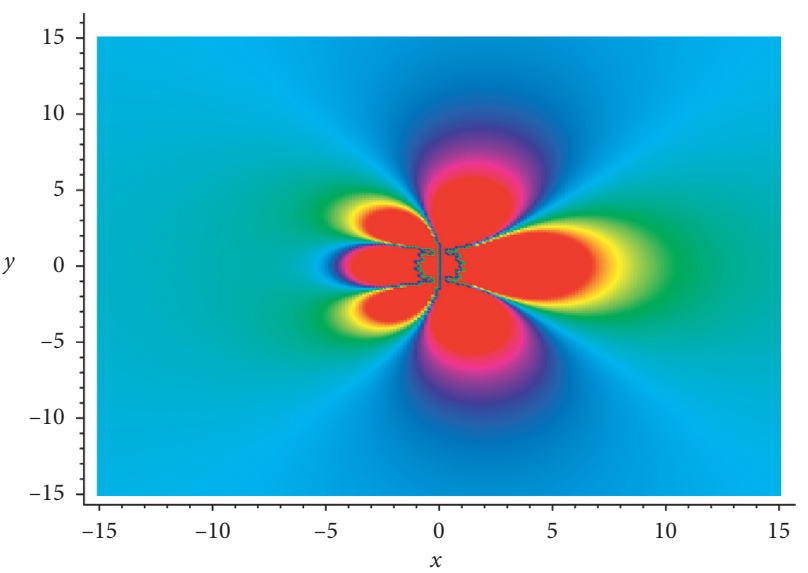

(c)

FIgURE 7: The density plot of the symmetry breaking lumps of the AB-KP systems (7a) and (7b), with the selected parameters $b_{1}$ and $b_{2}$ are (a) $b_{1}=b_{2}=0$ and (b) and (c) $b_{1}=b_{2}=10$, at time $t=0$.

where the functions $g$ and $h$ are given as follows:

$$
\begin{aligned}
& g=a_{1}\left(x-\frac{x_{0}}{2}\right)+a_{2} y+\frac{a_{1} c\left(a_{2}^{2}-2 a_{4}^{2}\right)}{2 a_{4}^{2}}\left(t-\frac{t_{0}}{2}\right), \\
& h=a_{4}\left(x-\frac{x_{0}}{2}\right)-\frac{a_{1} a_{2}}{a_{4}} y+\frac{c\left(a_{2}^{2}-2 a_{4}^{2}\right)}{2 a_{4}}\left(t-\frac{t_{0}}{2}\right),
\end{aligned}
$$

with $c, a_{1}, a_{2}, a_{4}, x_{0}$, and $t_{0}$ being some free real constants. Obviously, this set of solution (45a) and (45b) represents solitary waves in the form of rational structures, which is a family of two-wave solutions with different velocities and directions due to the linear functions $g$ and $h$ of the three variables $x, y$, and $t$.

If the constants are taken as $a_{1}=a_{4}=-1, a_{2}=c=$ $\alpha=1, \beta=1 / 6$, and $x_{0}=t_{0}=0$, equation (43) becomes

$$
f=f_{l}=\left(x-y-\frac{t}{2}\right)^{2}+\left(x+y-\frac{t}{2}\right)^{2}-2,
$$

according to equation (44). Figure 7 is a density plot of the lumps by equation (36) under the abovementioned parameter selection. Figure $7(\mathrm{a})$ is a normal first-order lump structure $\left(b_{1}=b_{2}=0\right)$ for the solution $A=B=-4\left(x^{2}-\right.$ $\left.y^{2}+1\right) /\left(x^{2}+y^{2}-1\right)^{2}$ at time $t=0$. Figures $7(\mathrm{~b})$ and $7(\mathrm{c})$ are two symmetry breaking lump structures for the solution $A, B=-4\left(x^{2}-y^{2}+1\right) /\left(x^{2}+y^{2}-1\right)^{2} \pm 20 x\left(x^{2}+3 x-3 y^{2}\right) /$ $\left(x^{2}+y^{2}-1\right)^{3}$, with the selected parameters $b_{1}=b_{2}=10$, at time $t=0$. As these solutions are all rational functions, the property of these functions describes symmetry breaking lump structures.

Furthermore, if we take $k_{i}=\delta_{i} \epsilon_{i}, \eta_{i 0}=\pi I, i=1,2,3,4$, and

$$
\begin{aligned}
& p_{1}=1+I, \\
& p_{2}=1-I, \\
& p_{3}=-p_{1}, \\
& p_{4}=-p_{2}, \\
& \delta_{3}=\delta_{1}, \\
& \delta_{4}=\delta_{2},
\end{aligned}
$$

after setting the limit $\epsilon_{i} \longrightarrow 0, i=1,2,3,4$, the function $f$ (26) can be simplified into 


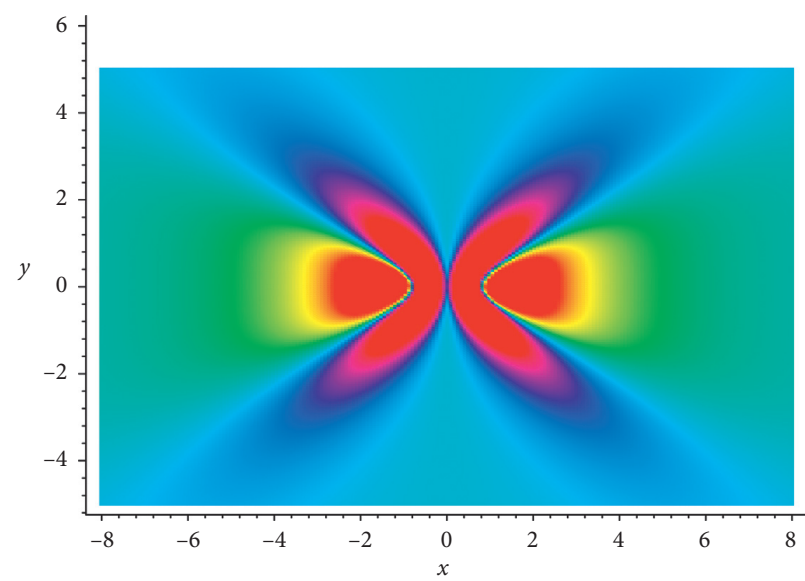

(a)

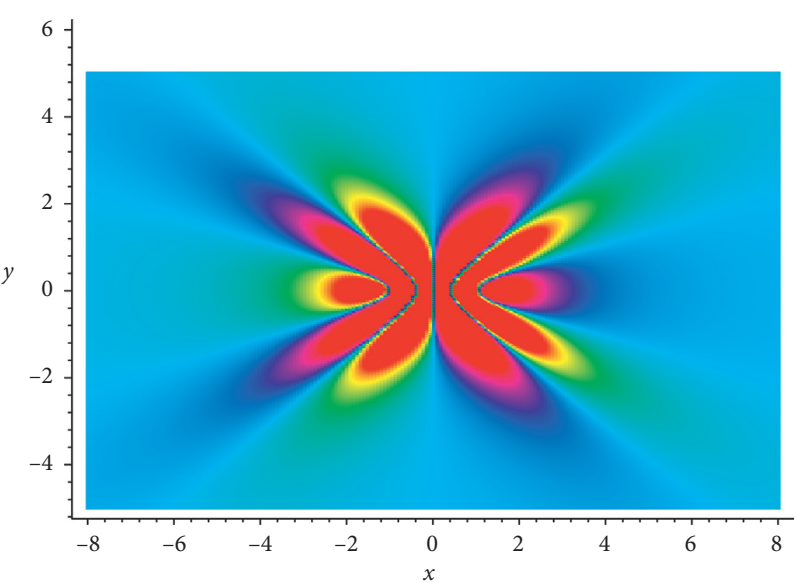

(b)

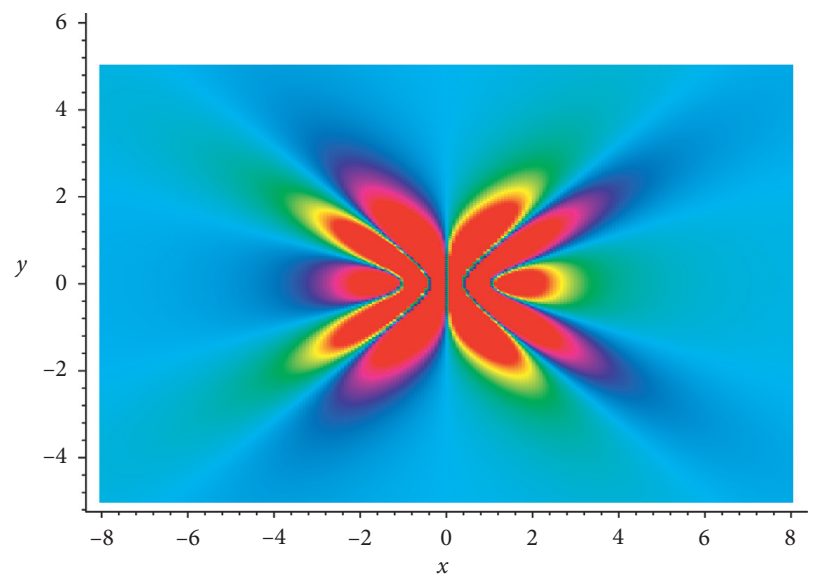

(c)

FIGURE 8: The symmetry breaking density plot of the second-order lumps of the AB-KP systems (7a) and (7b), with the selected parameters $b_{1}$ and $b_{2}$ are (a) $b_{1}=b_{2}=0$ and (b) and (c) $b_{1}=b_{2}=5$, at time $t=0$.

$$
\begin{aligned}
f= & f_{l}=c^{2}\left[\left(x-\frac{x_{0}}{2}\right)^{4}+4 y^{4}+4 c^{4}\left(t-\frac{t_{0}}{2}\right)^{4}\right] \\
& -4 c^{3}\left(x-\frac{x_{0}}{2}\right)^{3}\left(t-\frac{t_{0}}{2}\right)+8 c^{4}\left(x-\frac{x_{0}}{2}\right)^{2}\left(t-\frac{t_{0}}{2}\right)^{2} \\
& -4 c^{2}\left(x-\frac{x_{0}}{2}\right)\left(t-\frac{t_{0}}{2}\right)\left[2 c^{3}\left(t-\frac{t_{0}}{2}\right)^{2}-2 c y^{2}-3 \beta\right] \\
& -4 c^{3}\left(2 c y^{2}+3 \beta\right)\left(t-\frac{t_{0}}{2}\right)^{2}-36 c \beta y^{2}+81 \beta^{2} .
\end{aligned}
$$

Figure 8 shows the second-order lump structures when $c=-4, \alpha=1, \beta=1 / 6$, and $x_{0}=t_{0}=\eta_{10}=\eta_{20}=0$, which are symmetry breaking.

\section{Conclusion}

As everyone knows, the two-place correlated physical events widely exist in the field of natural science, and the discussed $\mathrm{AB}$ physics (two-place physics) has a profound influence on other scientific fields. In this work, by establishing a special
$\mathrm{AB}-\mathrm{KP}$ system via the parity with a shift of the space variable $x$ and time reversal with a delay, some group invariant solutions, such as symmetry breaking soliton, symmetry breaking breather, and symmetry breaking lump solutions have been presented through introducing an extended Bäcklund transformation and the established Hirota bilinear form. At the same time, the corresponding symmetry breaking structures of these explicit solutions are depicted according to the ansatz functions.

In fact, these are the following few open problems. Firstly, we may investigate more local and nonlocal symmetry breaking structures, such as the cnoidal wave and rogue wave through expression (2). Secondly, the arbitrary function $G(A, B)$ of $A$ and $B$ (which should be $\widehat{P}_{s}^{x} \widehat{T}_{d}$ invariant) is diverse, although we take $G(A, B)=(\alpha / 2)\left(A_{x}^{2}+\right.$ $\left.A A_{x x}-B_{x}^{2}-B B_{x x}\right)$ in this paper. Thirdly, algebraic structures involving the related Lie point symmetry and LieBäcklund symmetry reductions, and Bäcklund transformations determined by residual symmetries may be discussed mathematically for the AB-KP systems (7a) and (7b). Finally, the $\widehat{P}_{s}^{x} \widehat{T}_{d}$ symmetry of this paper could be generalized to other nonlinear systems by taking the specific elements of the larger $\widehat{P}_{s} \widehat{T}_{d} \widehat{C}$ symmetry group [15]. 


\section{Data Availability}

The data used to support the findings of this study are included within the article. For more details, the data are available from the corresponding author upon request.

\section{Conflicts of Interest}

The authors declare that they have no conflicts of interest.

\section{Acknowledgments}

The project was supported by the National Natural Science Foundation of China (nos. 11775104 and 11705077) and Scientific Research Foundation of the First-Class Discipline of Zhejiang Province (B) (no. 201601).

\section{References}

[1] W.-X. Ma, "The inverse scattering transform and soliton solutions of a combined modified Korteweg-de Vries equation," Journal of Mathematical Analysis and Applications, vol. 471, no. 1-2, pp. 796-811, 2019.

[2] W.-M. Ma, "Darboux transformations for a Lax integrable system in $2 n$-dimensions," Letters in Mathematical Physics, vol. 39, no. 1, pp. 33-49, 1997.

[3] W.-X. Ma and Y.-J. Zhang, "Darboux transformations of integrable couplings and applications," Reviews in Mathematical Physics, vol. 30, no. 2, p. 1850003, 2018.

[4] M. J. Ablowitz, A. Ramani, and H. Segur, "A connection between nonlinear evolution equations and ordinary differential equations of P-type. I, II," Journal of Mathematical Physics, vol. 21, no. 4, pp. 715-721, 1980.

[5] J. Weiss, "Bäcklund transformation and the Painlevé property," Journal of Mathematical Physics, vol. 27, no. 5, pp. 1296-1305, 1986.

[6] M. Musette and R. Conte, "Algorithmic method for deriving Lax pairs from the invariant Painlevé analysis of nonlinear partial differential equations," Journal of Mathematical Physics, vol. 32, no. 6, pp. 1450-1457, 1991.

[7] R. Hirota, "Exact solution of the Korteweg-de Vries equation for multiple collisions of solitons," Physical Review Letters, vol. 27, no. 18, pp. 1192-1194, 1971.

[8] R. Hirota, The Direct Method in Soliton Theory, Cambridge University Press, Cambridge, UK, 2004.

[9] W. X. Ma, "Generalized bilinear differential equations," Studies in Nonlinear Sciences, vol. 2, pp. 140-144, 2011.

[10] M. J. Ablowitz and Z. H. Musslimani, "Integrable nonlocal nonlinear Schrödinger equation," Physical Review Letters, vol. 110, no. 6, Article ID 064105, 2013.

[11] S. Y. Lou, "Alice-Bob systems, $\widehat{P}-\widehat{T}-\widehat{C}$ symmetry invariant and symmetry breaking soliton solutions," Journal of Mathematical Physics, vol. 59, no. 8, Article ID 083507, 2018.

[12] S. Y. Lou, "Alice-Bob systems, $P_{s}-T_{d}-C$ principles and multi-soliton solutions," 2016, https://arxiv.org/abs/1603. 03975.

[13] C. Li, S. Y. Lou, and M. Jia, "Coherent structure of Alice-Bob modified Korteweg de-Vries equation,” Nonlinear Dynamics, vol. 93, no. 4, pp. 1799-1808, 2018.

[14] S. Y. Lou, "Prohibitions caused by nonlocality for nonlocal Boussinesq-KdV type systems," Studies in Applied Mathematics, vol. 143, no. 2, pp. 123-138, 2019.
[15] S. Y. Lou, "Multi-place nonlocal systems," 2019, https://arxiv. org/abs/1901.02828.

[16] M. Jia and S. Y. Lou, "Exact $P_{s} T_{d}$ invariant and $P_{s} T_{d}$ symmetric breaking solutions, symmetry reductions and Bäklund transformations for an $\mathrm{AB}-\mathrm{KdV}$ system," Physics Letters A, vol. 382, no. 17, pp. 1157-1166, 2018.

[17] S.-Y. Lou and Z.-J. Qiao, "Alice-Bob peakon systems," Chinese Physics Letters, vol. 34, no. 10, Article ID 100201, 2017.

[18] S. Y. Lou and F. Huang, "Alice-Bob physics: coherent solutions of nonlocal KdV systems," Scientific Reports, vol. 7, no. 1, 2017.

[19] Z.-Y. Ma, J.-X. Fei, and J.-C. Chen, "Nonlocal symmetry and explicit solution of the Alice-Bob modified Korteweg-de Vries equation," Communications in Theoretical Physics, vol. 70, no. 1, pp. 031-037, 2018.

[20] S. Y. Lou, "From nothing to something II: nonlinear systems via consistent correlated bang," Chinese Physics Letters, vol. 34, no. 6, Article ID 060201, 2017.

[21] X.-Y. Tang, Z.-F. Liang, Z. F. Liang, and J. Y. Wang, "A general nonlocal variable coefficient $\mathrm{KdV}$ equation with shifted parity and delayed time reversal," Nonlinear Dynamics, vol. 92, no. 3, pp. 815-825, 2018.

[22] X.-Y. Tang, Z.-F. Liang, and X.-Z. Hao, "Nonlinear waves of a nonlocal modified $\mathrm{KdV}$ equation in the atmospheric and oceanic dynamical system," Communications in Nonlinear Science and Numerical Simulation, vol. 60, pp. 62-71, 2018.

[23] D.-J. Ding, D.-Q. Jin, and C.-Q. Dai, "Analytical solutions of differential-difference sine-Gordon equation," Thermal Science, vol. 21, no. 4, pp. 1701-1705, 2017.

[24] Y.-Y. Wang, C.-Q. Dai, Y.-Q. Xu, J. Zheng, and Y. Fan, "Dynamics of nonlocal and localized spatiotemporal solitons for a partially nonlocal nonlinear Schrodinger equation," Nonlinear Dynamics, vol. 92, no. 3, pp. 1261-1269, 2018.

[25] C.-Q. Dai, Y.-Y. Wang, Y. Fan, and D.-G. Yu, "Reconstruction of stability for Gaussian spatial solitons in quintic-septimal nonlinear materials under PT-symmetric potentials," Nonlinear Dynamics, vol. 92, no. 3, pp. 1351-1358, 2018.

[26] M. J. Ablowitz and H. Segur, Solitons and the Inverse Scattering Transform, SIAM, Philadelphia, PA, USA, 1981.

[27] V. I. Petviashvili and O. V. Pokhotelov, Solitary Waves in Plasmas and in the Atmosphere, Energoatomizdat, Moscow, Russia, 1989.

[28] G. C. Das and J. Sarma, "Evolution of solitary waves in multicomponent plasmas," Chaos, Solitons \& Fractals, vol. 9, no. 6, pp. 901-911, 1998.

[29] Z. D. Dai, Z. J. Liu, and D. L. Li, "Exact periodic solitary-wave solution for KdV equation," Chinese Physics Letters, vol. 25, no. 5, pp. 1531-1533, 2008.

[30] C. Wang, H. Fang, and X. Tang, "State transition of lump-type waves for the $(2+1)$-dimensional generalized KdV equation," Nonlinear Dynamics, vol. 95, no. 4, pp. 2943-2961, 2019. 